\title{
GANGLIONIC AND NON-GANGLIONIC NEURONS IN FROG HEART: DO THEY DIFFER?
}

\author{
Darius Batulevičius, Gertrūda Skripkiené, \\ Greta Graužinytė, Augustina Grigaitė, Valdas Skripka \\ Institute of Anatomy, Faculty of Medicine, \\ Lithuanian University of Health Sciences, Kaunas, Lithuania
}

\begin{abstract}
This study was designed to compare the morphology of neurons in relation to their distance from the major nerve trunks in the heart of the frog Rana temporaria. Seventy-nine intracardiac neurons were labelled intracellularly with fluorescent markers Lucifer Yellow $\mathrm{CH}$ and Alexa Fluor 568. The neurons located on the extensions of the vagus nerve were considered as ganglionic, while neurons spread loosely at further distance from these extensions were considered as non-ganglionic. The mean area of the soma in ganglionic neurons was about 25\% larger than in non-ganglionic neurons. Ganglionic neurons had a higher soma area/nucleus area ratio than non-ganglionic neurons. Although both the total number and the total length of dendrite-like processes was similar between the two groups, ganglionic neurons had significantly fewer dendrite-like processes from the soma (1.5 \pm 0.3 vs. $3.9 \pm 1.0$; $\mathrm{P}<0.05)$ and shorter total length of these processes from the soma $(63 \pm 18 \mu \mathrm{m}$ vs. $178 \pm 51 \mu \mathrm{m} ; \mathrm{P}<0.05)$. In conclusion, ganglionic and non-ganglionic frog intracardiac neurons exhibit substantial morphological differences. We hypothesize that these differences may indicate different projections or variations in the number of their preganglionic inputs.
\end{abstract}

Keywords: axon; dendrites; soma; heart; autonomic; ganglion 


\section{INTRODUCTION}

Dendritic and soma morphology is of pivotal importance in neurosciences, because it is indicative of synaptic connections and functional properties of neurons. It has been shown in a number of mammalian models that the number of primary dendrites of an autonomic neuron correlates well to the number of presynaptic inputs $[12,13]$. Autonomic neurons that receive only one presynaptic input usually have no primary dendrites. The number of primary dendrites increases with the number of presynaptic inputs [12, 13]. According to earlier studies, cranial and terminal autonomic ganglia of small vertebrates like the frog, the mouse or the hamster contain mostly non-dendritic neurons that are innervated by $1-2$ preganglionic axons $[2,13]$. The complexity of the dendrites and the soma of autonomic neurons increases with the size of the species [13] or with closer distance to their innervation targets [5].

Although the structure and distribution of frog intracardiac neurons has been described recently [1], so far there are no studies available on the morphology of frog intracardiac neurons in relation to their position in the heart. Most of frog intracardiac neurons are distributed along the extensions of vagus nerves into the venal sinus, the interatrial septum and the atrio-ventricular region [1]. Historically, the accumulations of neurons along the extensions of vagus nerves into the frog venal sinus, interatrial septum and atrio-ventricular region have been termed as Remak's, Ludwig's and Bidder's ganglia. However, it was also shown recently that a considerable proportion of frog intracardiac neurons is not accumulated above ganglia but is located at a further distance from the extensions of vagus nerves [1]. The structure and function of these non-ganglionic neurons is not yet known.

The current study was designed to compare the morphology of ganglionic and non-ganglionic neurons in the heart of the frog Rana temporaria. We report that ganglionic and non-ganglionic frog intracardiac neurons exhibit substantial morphological differences.

\section{MATERIAL AND METHODS}

The study was performed on 13 adult (20-35 g in weight) common frogs (Rana temporaria). This investigation conforms to both the "Principles of laboratory animal care" (NIH publication No 86-23, revised 1985) and the local guidelines for the use of experimental animals. The frogs were euthanized by decapitation. 


\section{Intracellular injection and microscopy of neurons}

Atria with an interatrial septum, venal sinus and atrio-ventricular region were dissected and pinned flat in a Petri dish with physiologic solution. Intracellular fluorescent dyes Lucifer Yellow CH (Aldrich, USA) and Alexa Fluor 568 (Molecular Probes, USA) were injected ionophoretically into the somata of neurons. The dyes were injected by applying $10 \mathrm{nA}$ constant negative current for 10-15 min with Microionophoresis Dual Current Generator 260 (World Precision Instruments, USA). Following injection, the tissue was fixed in $4 \%$ paraformaldehyde in phosphate-buffered saline (PBS) overnight, rinsed in PBS for $30 \mathrm{~min}$ and mounted on glass slides using the Vectashield mounting medium (Vectorlabs, USA). The neurons were observed at $400 \times$ magnification by the fluorescent microscope Imager Z1 (Zeiss, Germany) equipped with an Apotome (Zeiss, Germany) and digital camera AxioCam MRm (Zeiss, Germany). We used the fluorescein isothiocyanate (FITC) and cyanine (Cy3) fluorescence filters to observe the Lucifer Yellow $\mathrm{CH}$ and Alexa Fluor 568 labelled neurons, respectively. Measurements and reconstructions of neurons were made with AxioVision 4.7 image analysing software (Zeiss, Germany).

\section{Criteria for ganglionic and non-ganglionic neurons, measurements}

A nerve cell was selected for our study if the injected intracellular dye filled its soma and revealed the neuronal processes. Forty-three neurons were classified as ganglionic neurons because their somata contacted major nerve trunks. Thirty-six neurons were classified as non-ganglionic neurons because their somata did not contact major nerve trunks and were spread loosely throughout the venal sinus, the interatrial septum and the atrio-ventricular region. The area of the neuronal soma and the length of neuronal processes were measured in extended focus images of neurons by AxioVision 4.7 software (Zeiss, Germany). Comparison of measurements was performed with Student $t$-test for independent samples (Origin 6.1, OriginLab, USA). Significance was accepted at $\mathrm{P}<0.05$.

\section{RESULTS}

\section{Soma}

Both long and short axes of neuronal soma were significantly longer in ganglionic neurons compared to non-ganglionic neurons (short axis: $34 \pm 1 \mu \mathrm{m}$ 
vs. $29 \pm 1$; long axis: $46 \pm 2$ vs. $39 \pm 2$; $<<0.005$ ). The mean area of the soma of ganglionic neurons was about $25 \%$ larger than in non-ganglionic neurons $\left(1286 \pm 74 \mu \mathrm{m}^{2}\right.$ vs. $\left.959 \pm 76 \mu \mathrm{m}^{2} ; \mathrm{P}<0.005\right)$. In addition, ganglionic neurons had a higher soma area/nucleus area ratio than non-ganglionic neurons $(6.6 \pm 0.2$ vs. $5.5 \pm 0.3 ; \mathrm{P}<0.005)$.

\section{Dendrite-like processes}

The total number of processes did not differ significantly between ganglionic and non-ganglionic neurons ( $9 \pm 1$ vs. $11 \pm 1 ; \mathrm{P}=0.18$ ). However, ganglionic neurons had fewer processes projecting from the soma ( $1.5 \pm 0.3$ vs. $3.9 \pm 1.0$; $\mathrm{P}<0.05$; Fig. 1). The number of processes projecting from the axon was very
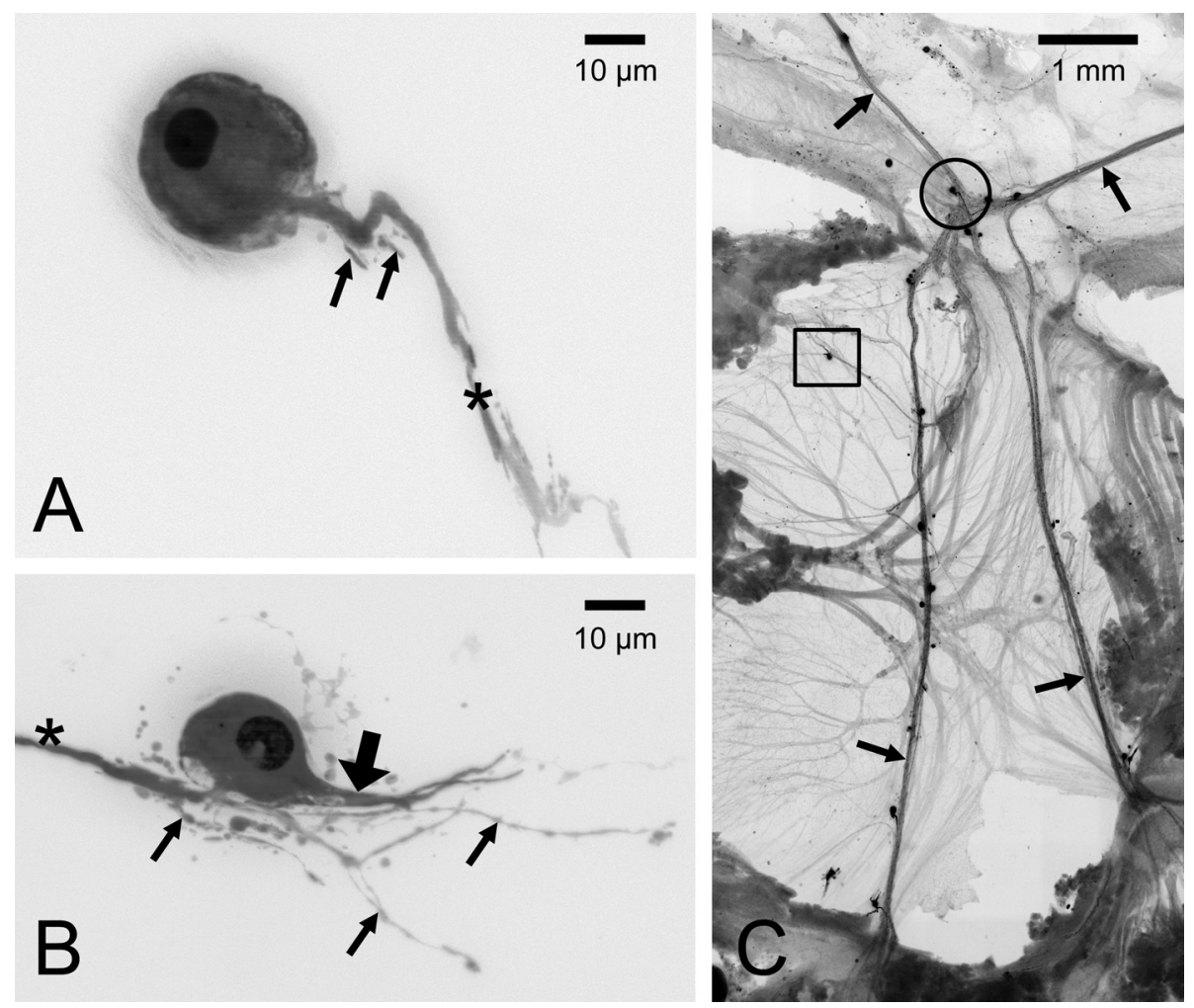

Figure 1. Ganglionic neuron (A) and non-ganglionic neuron (B) in frog heart. Asterisks mark axons. Thin arrows point to dendrite-like processes from the axon. Thick arrow in $B$ points to a dendrite-like process from the soma. Panel $C$ shows frog interatrial septum with injected neurons at low magnification. Arrows in $C$ point to extensions of vagus nerves. Circular area marks injected neuron that was classified as ganglionic. Rectangle area marks injected neuron that was classified as non-ganglionic. 


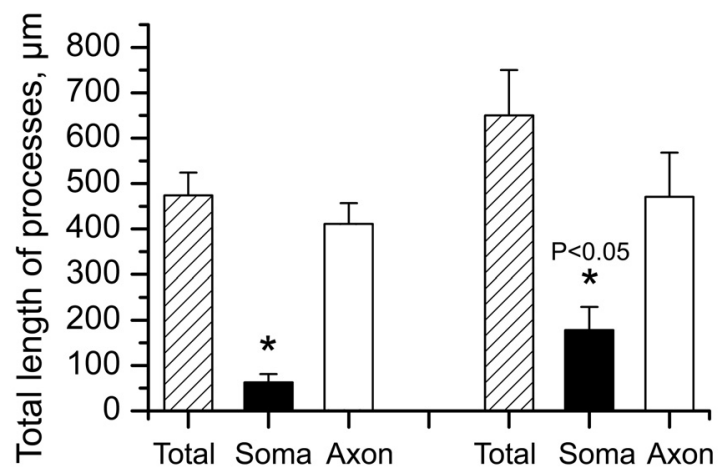

Ganglionic neurons Non-ganglionic neurons
Figure 2. Total length of dendrite-like processes (in $\mu \mathrm{m}$ ) of ganglionic neurons $(n=43)$ and nonganglionic neurons $(n=36)$ in frog heart. Total length of processes from soma is significantly different at $\mathrm{P}<0.05$.

similar between ganglionic and non-ganglionic neurons ( $7.4 \pm 0.8$ vs. $6.9 \pm 0.7$; $\mathrm{P}=0.67$ ). Although the total length of processes did not differ between ganglionic and non-ganglionic neurons $(474 \pm 50 \mu \mathrm{m}$ vs. $650 \pm 100 \mu \mathrm{m} ; \mathrm{P}=0.11)$, ganglionic neurons had a shorter total length of processes projecting from the soma $(63 \pm 18 \mu \mathrm{m}$ vs. $178 \pm 51 \mu \mathrm{m} ; \mathrm{P}<0.05$; Fig. 2$)$. The total length of processes projecting from the axon was similar between ganglionic and non-ganglionic neurons $(411 \pm 46 \mu \mathrm{m}$ vs. $471 \pm 97 \mu \mathrm{m} ; \mathrm{P}=0.56)$.

\section{DISCUSSION}

The findings of this study suggest that frog intracardiac neurons positioned in ganglia on extensions of vagus nerves differ structurally from neurons that are located at a further distance from these major nerve trunks. Nonganglionic neurons have a smaller soma, more dendrite-like processes from the soma and less cytoplasm than ganglionic neurons. Although the reasons of structural heterogeneity between ganglionic and non-ganglionic neurons are still unknown, we hypothesize below that these differences might imply 1) differences in their innervation projections or 2) variations in the number of presynaptic inputs.

Earlier study on frog intracardiac neurons has shown that neurons located on major nerve trunks send their axons into ventricles, while neurons located at a further distance from major nerve trunks send their axons towards atria [1]. Thus, it is likely that ganglionic neurons that have comparatively large somata and a higher soma area/nucleus area ratio send their axons for more distant targets compared to non-ganglionic neurons. On the other hand, non-ganglionic 
neurons that have comparatively more dendrite-like processes from the soma may be involved in the innervation of targets in proximity to their somata. This assumption is partly supported by the study on guinea pig paratracheal neurons where the soma morphology of neurons lying in the ganglionated plexus on trachealis muscle was more complex than of those positioned in more distant associated nerve trunks [5].

Frog intracardiac neurons are typically innervated by $1-2$ preganglionic axons [2]. Only about $8 \%$ of frog intracardiac neurons receive more than two presynaptic inputs [2]. From previous studies on mammalian heart it is also known that neurons without dendrites are frequently innervated by a single axon, while those with dendrites are innervated by multiple axons proportionally to the structural complexity of dendrites [12]. Thus, finding more dendrite-like processes on the somata of non-ganglionic neurons may indicate the possibility that these neurons receive more synaptic inputs compared to ganglionic neurons.

With respect to the function of frog intracardiac neurons, it is still accepted that all intracardiac neurons in frogs utilize acetylcholine as a neurotransmitter and serve a parasympathetic function [3]. Although somata of catecholaminergic and peptidergic neurons have been reported in the mudpuppy, Necturus maculosus [10, 11], there is no evidence yet on non-cholinergic neuronal somata in the heart of anuran amphibians. Nonetheless, it has been shown previously in mammalian autonomic ganglia that the population of cholinergic neurons is not uniform, as many of those neurons express additional transmitters and differ electrophysiologically [7, 9]. Considerable heterogeneity has been reported in the morphological, neurochemical, and electrophysiological properties of neurons in the sympathetic ganglia $[4,6]$. Relationships between the shape and electrophysiological characteristics of neurons in the intestine of the guinea pig have been proposed [8], but there are also reports that dendritic complexity does not necessarily discriminate between functional types of autonomic neurons [6, 7]. E.g., in the guinea pig pancreas, tonic cells were morphologically indistinguishable from phasic cells [7]. As similar studies on frog intracardiac neurons are still lacking, more work should be done in correlating morphological, neurochemical and electrophysiological data on frog intracardiac neurons. 


\section{REFERENCES}

1. Batulevicius D., Skripkiene G., Batuleviciene V., Skripka V., Dabuzinskiene A., Pauza D. H. (2012). Distribution, structure and projections of the frog intracardiac neurons. Auton Neurosci, 168, 1-2, 14-24. https://doi.org/10.1016/j.autneu.2012.01.004

2. Dennis M.J., Sargent P.B. (1978). Multiple innervation of normal and re-innervated parasympathetic neurones in the frog cardiac ganglion. J Physiol, 281, 63-75. https://doi.org/10.1113/jphysiol.1978.sp012409

3. Gibbins I.L., Morris J.L. (2006). Structure of peripheral synapses: autonomic ganglia. Cell Tissue Res, 326, 205-220. https://doi.org/10.1007/s00441-006-0233-1

4. Jobling P., Gibbins I.L. (1999). Electrophysiological and morphological diversity of mouse sympathetic neurons. J Neurophysiol, 82, 2747-2764.

5. Lees G.M., Pacitti E.G., Mackenzie G.M. (1997). Morphology and electrophysiology of guinea-pig paratracheal neurones. Anat Rec, 247, 261-270. https:// doi.org/10.1002/(SICI)1097-0185(199702)247:2<261::AID-AR13>3.0.CO;2-N

6. Li C., Horn J.P. (2006). Physiological classification of sympathetic neurons in the rat superior cervical ganglion. J Neurophysiol, 95, 187-195. https://doi.org/10.1152/jn.00779.2005

7. Liu M.T., Kirchgessner A.L. (1997). Guinea pig pancreatic neurons: morphology, neurochemistry, electrical properties, and response to 5-HT. Am J Physiol, 36, G1273-G1289.

8. Lomax A.E., Sharkey K.A., Bertrand P.P., Low A.M., Bornstein J.C., Furness J.B. (1999). Correlation of morphology, electrophysiology and chemistry of neurons in the myenteric plexus of the guinea-pig distal colon. J Auton Nerv Syst, 76, 45-61. https://doi.org/10.1016/S0165-1838(99)00008-9

9. Luna F., Barrientos E., Alatriste V., Martinez I., Limon I.D., Gonzalez-Flores O. (2015). Morphology and chemical phenotype of the ovarian intrinsic neurons in neonate and sexually mature reproductive guinea pig. Adv Reprod Sci, 3, 13-26. https://doi.org/10.4236/arsci.2015.31002

10. McMahan U.J., Purves D. (1976). Visual identification of two kinds of nerve cells and their synaptic contacts in a living autonomic ganglion of the mudpuppy (Necturus maculosus). J Physiol, 405-425. https://doi.org/10.1113/jphysiol.1976.sp011238

11. Parsons R.L., Neel D.S., McKeon T.W., Carraway R.E. (1987). Organization of a vertebrate cardiac ganglion: a correlated biochemical and histochemical study. J Neurosci, 7, 837-846.

12. Purves D., Hume R.I. (1981). The relation of postsynaptic geometry to the number of presynaptic axons that innervate autonomic ganglion cells. J Neurosci, 1, $441-452$. 
13. Snider W.D. (1987). The dendritic complexity and innervation of submandibular neurons in five species of mammals. J Neurosci, 7, 1760-1768.

\section{Address for correspondence:}

Darius Batulevičius

Institute of Anatomy

Faculty of Medicine

Lithuanian University of Health Sciences

A. Mickevičiaus 9, LT-44307 Kaunas, Lithuania

E-mail: darius.batulevicius@lsmuni.lt; phone: +370 37327295 